\title{
Membrane-bound molecular rotors measure viscosity in live cells via fluorescence lifetime imaging
}

James A. Levitt, ${ }^{1}$ Marina K. Kuimova, ${ }^{2 *}$ Gokhan Yahioglu, ${ }^{2,3 *}$ Pei-Hua Chung, ${ }^{1}$ Klaus Suhling, ${ }^{1 *}$ and David Phillips ${ }^{2}$

${ }^{1}$ Department of Physics, King's College London, Strand, London WC2R 2LS, UK.

${ }^{2}$ Department of Chemistry, Imperial College London, Exhibition Road, London SW7 2AZ, UK.

${ }^{3}$ PhotoBiotics Ltd, 21 Wilson Street, London EC2M 2TD, UK.

m.kuimova@imperial.ac.uk; g.yahioglu@imperial.ac.uk; klaus.suhling@kcl.ac.uk

RECEIVED DATE (to be automatically inserted after your manuscript is accepted if required according to the journal that you are submitting your paper to) 


\begin{abstract}
We report intracellular fluorescence lifetime imaging (FLIM) and fluorescence anisotropy measurements of two meso-substituted fluorophores based on the boron-dipyrrin (BODIPY) structure. Both dyes incorporate hydrophobic groups, which render them membrane-soluble. We have obtained quantum yields, radiative and non-radiative rate constants, fluorescence lifetimes and time-resolved fluorescence anisotropy of the dyes in homogeneous methanol/glycerol solutions of varying viscosity from $0.6 \mathrm{cP}$ to $950 \mathrm{cP}$. We find that the fluorescence lifetimes and rotational correlation times for both dyes increase with increasing viscosity, as predicted by theory. These molecules can thus serve as fluorescent molecular rotors to report on local microviscosity, including that in live cells. The dyes are readily taken up by cells as imaged using confocal fluorescence microscopy. Using FLIM we have detected two distinct fluorescence lifetime populations for both dyes in live SK-OV-3 human ovarian carcinoma cells, corresponding to apparent viscosity values of $160 \pm 20 \mathrm{cP}$ and $260 \pm 40 \mathrm{cP}$, each found in distinct intracellular domains. In both cellular domains, independent of the fluorophore used, the viscosity values significantly exceed that expected for the aqueous phase of cellular cytoplasm, suggesting slower diffusion and reaction rates in this hydrophobic microenvironment. FLIM measurements were complemented with time-resolved fluorescence anisotropy measurements, which confirm the high viscosity values in the immediate environment of both rotors. The present study highlights the power of FLIM to map heterogeneous microenvironments of complex biological systems and also the use of fluorescent molecular rotors as microviscosity sensors.
\end{abstract}




\section{Introduction}

Diffusion is of fundamental importance in the physical and life sciences. Diffusion is often an important rate-determining step in chemical reactions or biological processes, and as such is expected to play a role in a wide range of intracellular events. For example, some signalling pathways along with protein-protein interactions are dependent on the transport of biomolecules within and between intracellular compartments. ${ }^{1}$ Knowledge of the physico-chemical local environment in these compartments will aid not only the understanding of intracellular reaction kinetics and mechanisms but can potentially assist in development and understanding of the mechanisms of targeted therapies for cancer. $^{2}$ It is thus of great interest to observe diffusion processes in cells. However, monitoring diffusion on a micrometer scale presents a significant challenge.

One major factor influencing the rate of diffusion in condensed media is the viscosity of the medium. On a macroscopic scale, viscosity is typically measured by mechanical methods. ${ }^{3}$ However, in order to monitor viscosity inside cells, it is necessary to use techniques which sample length scales of the order of the dimensions of intracellular compartments, i.e. micrometers. This can be accomplished using optical techniques by employing a variety of fluorescent probes and markers. Optical techniques allow probing of microscopic objects, are minimally invasive and thus can be successfully applied to biological tissue and even live cells. In an optical experiment, one can obtain information related to interactions of a probe with its environment and hence probe the microstructure of the sample.

Amongst the techniques for measuring diffusion and viscosity based on fluorescence microscopy are single molecule tracking, ${ }^{4-6}$ fluorescence recovery after photobleaching (FRAP) ${ }^{7,8}$, fluorescence correlation spectroscopy $(\mathrm{FCS})^{9,10}$ and raster imaging correlation spectroscopy (RICS) ${ }^{11-13}$ which can probe the translational diffusion of labelled molecules. The diffusion rate can also be estimated by measuring diffusion-limited processes in cells, for example formation and decay of cytotoxic singlet 
molecular oxygen, ${ }^{2,14}$ and viscosity-dependent singlet oxygen quenching reactions within cells. ${ }^{15}$ Furthermore, rotational diffusion can be measured via time-resolved fluorescence anisotropy measurements, which, combined with imaging, allows mapping of the rotational diffusion (TRFAIM). ${ }^{16-21}$ Another technique for measuring microviscosity, particularly that of a biological environment, is fluorescence imaging of molecular rotors. ${ }^{2,22-27}$

Molecular rotors are fluorophores whose fluorescence lifetime changes as a function of the viscosity of their microenvironment. ${ }^{22}$ Their radiative de-excitation pathway competes with intramolecular twisting, which leads to non-radiative deactivation of the excited state. The rate constant for the latter pathway is slowed down in viscous media, such that the fluorescence lifetime and quantum yield are high in viscous microenvironments, and low in non-viscous microenvironments. ${ }^{22}$ Molecular rotors have been highlighted as promising candidates for measurements of local viscosity using the change of fluorescence quantum yield. ${ }^{22}$ The main problem in this approach is distinguishing between viscosity and other factors which might affect the fluorescence intensity, such as the local concentration of the fluorophore or local variations in the optical properties of the medium (e.g. refractive index). A ratiometric approach, using probes that incorporate two linked independent fluorophores, has been suggested to solve these problems. ${ }^{2,23,28}$ In this approach the fluorescence intensity of one of the two fluorophores is not affected by viscosity and is used to determine the concentration, while the other acts as a molecular rotor. In this way the precise calibration of the viscosity in fluorescence intensity measurements is possible and has been demonstrated.,23,28 Alternatively, one can exploit the fluorescence lifetime of molecular rotors, which changes along with fluorescence quantum yield when the non-radiative decay contribution changes as a function of viscosity. ${ }^{24}$ In order to obtain maps of viscosity in heterogeneous environments, fluorescence lifetime imaging (FLIM) of molecular rotors can be used. FLIM combines the benefits of measuring the fluorescence lifetime, which is highly sensitive to environmental parameters and independent of fluorophore concentration, with the high spatial and temporal resolution afforded by fluorescence microscopy with ultrafast excitation sources and photon 
counting detection. ${ }^{29,30}$ We have recently shown that this approach allows straightforward calibration and affords direct mapping of the microviscosity in live cells. ${ }^{24}$ Fluorescence decay measurements of molecular rotors do not require conjugation of the molecular rotor to another fluorescence label. This often means a more facile synthesis of the probe and, in addition, leaves the spectral window vacant (due to the lack of the second reference fluorophore) for use with additional optical probes. ${ }^{31}$

Recently we have reported that local microviscosity in distinct organelles of single live cells can be as high as $140 \mathrm{cP}, 2,24$ which is $c a .100$ times higher than that expected from the aqueous environment of cell cytoplasm, 1-3 cP..$^{28,32,33}$ Independent measurements of diffusion and reactions of short lived cytotoxic species, singlet molecular oxygen in single cells ${ }^{2,14,15}$ also indicated that (i) diffusion rate inside cellular organelles can be very slow and (ii) viscosity inside cells is highly heterogeneous. Additionally, viscosity can change as a result of various intracellular processes, such as cell death. ${ }^{2}$ These results emphasize the need to be able to map the heterogeneous environment of the cell with submicrometer spatial resolution, in real time, to enable monitoring the progress and dynamics of the intracellular processes.

Here we have used FLIM and polarization-resolved fluorescence decay measurements of two structurally related molecular rotors based on meso-substituted BODIPY (Scheme 1) which readily incorporate into live cancer cells. Long hydrophobic tails have been designed to render our probes membrane-soluble and hence report on the membrane and other hydrophobic domains in live cells. These dyes are extremely well suited for use as probes of biological environments, e.g. cells, as their optimum excitation and emission wavelengths are in the visible region thus reducing the possibility of photodamage and phototoxicity. 

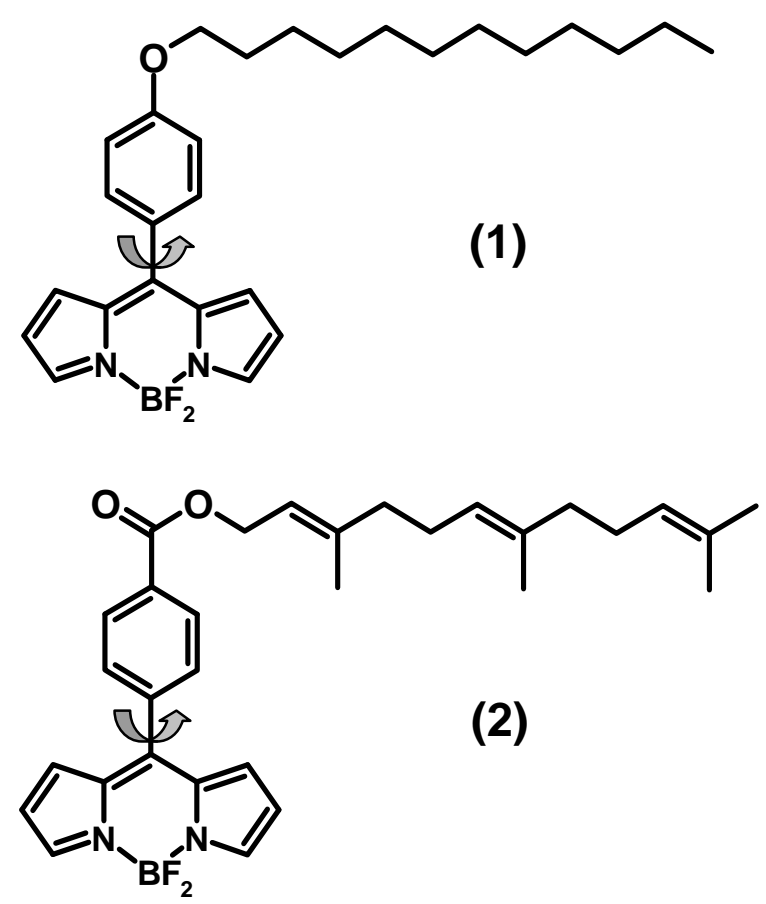

Scheme 1. The chemical structure of the two meso-substituted boron-dipyrrin (BODIPY) dyes used in this study.

\section{Theoretical Background}

The fluorescence quantum yield $\Phi_{f}$ and fluorescence lifetime $\tau_{f}$ of molecular rotors as a function of viscosity $\eta$ is described well by the Förster - Hoffmann equation (1) ${ }^{34}$

$$
\Phi_{f}=z \eta^{\alpha}
$$

where

$$
\Phi_{f}=\frac{k_{r}}{k_{r}+k_{n r}}
$$

such that

$$
\tau_{f}=z k_{r}^{-1} \eta^{\alpha}
$$

as

$$
\tau_{f}=\frac{1}{k_{r}+k_{n r}}
$$


where $k_{r}$ is the radiative rate constant, $k_{n r}$ is the non-radiative rate constant, and $\mathrm{z}$ and $\alpha$ are constants. ${ }^{35}$ In a photon-counting confocal FLIM experiment the radiation from a pulsed excitation source is scanned across the sample and the fluorescence photon arrival times typically collected by time-gating electronics ${ }^{36}$ or time-correlated single-photon counting. ${ }^{37,38}$ The fluorescence decay in each pixel of the image is fitted to an exponential decay, from which a value for $\tau_{f} \quad$ can be extracted. Taking the $\log$ arithm of equation (3), a plot of $\log \tau_{f} \quad$ versus $\log \eta$ should yield a straight line according to

$$
\log \tau_{f}=\alpha \log \eta+\log \left(\frac{z}{k_{r}}\right)
$$

which serves as a calibration that allows conversion of the fluorescence lifetime $\tau_{f}$ into viscosity $\eta$.

Upon excitation with linearly polarized light, rotational diffusion of the fluorophore in its excited state results in a depolarization of the fluorescence. ${ }^{35,39}$ Time-resolved fluorescence anisotropy imaging measures fluorescence decays at polarizations parallel and perpendicular to that of the polarization vector of the exciting light. The time-resolved fluorescence anisotropy, $r(t)$, can be defined as

$$
r(t)=\frac{I_{\|}(t)-G I_{\perp}(t)}{I_{\|}(t)+2 G I_{\perp}(t)}
$$

where $I_{\|}(t)$ and $I_{\perp}(t)$ are the fluorescence intensity decays parallel and perpendicular to the polarization vector of the exciting light. The term $G$ accounts for different transmission and detection efficiencies of the imaging system for parallel and perpendicular polarization. ${ }^{16-18,20,35,39}$

For a spherical molecule, $r(t)$ decays as a single exponential and is related to the rotational correlation time, $\theta$, according to

$$
r(t)=\left(r_{0}-r_{\infty}\right) \exp \left(-\frac{t}{\theta}\right)+r_{\infty}
$$


where $r_{0}$ is the initial anisotropy and $r_{\infty}$ is the limiting anisotropy which accounts for a restricted

rotational mobility. ${ }^{35}$ For a spherical molecule in an isotropic medium, $\theta$ is directly proportional to the viscosity, $\eta$, of the solvent and the molecular hydrodynamic volume, $v$, of the rotating molecule:

$$
\theta=\left(6 D_{r o t}\right)^{-1}=\frac{\eta v}{k_{b} T}
$$

where $D_{r o t}$ is the rotational diffusion constant, $k_{b}$ is the Boltzmann constant and $T$ is the absolute temperature.

\section{Materials and methods}

\section{Synthesis of meso-substituted BODIPY dyes 1 and 2}

\section{Synthesis and characterization of 1 .}

Compound 1 was synthesized by the method of Wagner and Lindsey. ${ }^{40}$ DDQ (280 mg, $\left.1.23 \mathrm{mmol}\right)$ was added to a $25 \mathrm{ml}$ solution of 5-(4-dodecyloxyphenyl)dipyrromethane (500 $\mathrm{mg}, 1.23 \mathrm{mmol})$ in toluene. The reaction mixture was kept in the dark and stirred at room temperature. Following 5 minutes of stirring, TLC (silica gel, 20\% ethyl acetate/hexane) showed the consumption of the starting dipyrromethane at which point triethylamine $(1.2 \mathrm{ml}, 8.61 \mathrm{mmol})$ was added followed immediately by boron trifluoride etherate $\left(1.1 \mathrm{ml}\right.$ of neat $\mathrm{BF}_{3}$-etherate, $\left.8.61 \mathrm{mmol}\right)$. The reaction mixture was stirred at room temperature for 1 hour, decanted from the dark green sludge which was washed with toluene (10 $\mathrm{ml})$ and the combined toluene extracts washed with water, dried $\left(\mathrm{Na}_{2} \mathrm{SO}_{4}\right)$ and evaporated to give a dark-green viscous oil. This was purified by column chromatography (silica gel, DCM, $\mathrm{R}_{\mathrm{f}} 0.54$ ) to give compound 1 as a red-orange crystalline solid. Yield $0.19 \mathrm{~g}(34 \%):{ }^{1} \mathrm{H}$ NMR $\left(400 \mathrm{MHz}, \mathrm{CDCl}_{3}\right) \delta 7.94$ (s, 2H, pyrrole-H); 7.55 (d, 2H, J = 8.6 Hz, phenyl-H); 7.05 (d, 2H, J = 8.6 Hz, phenyl-H); 7.01 (d, 2H, J $=4.1$, pyrrole- $\mathrm{H}) ; 6.57(\mathrm{~m}, 2 \mathrm{H}$, pyrrole- $\mathrm{H}) ; 4.07\left(\mathrm{t}, 2 \mathrm{H}, \mathrm{J}=6.5 \mathrm{~Hz}, \mathrm{O}-\mathrm{CH}_{2}\right) ; 1.86\left(\mathrm{~m}, 2 \mathrm{H}, \mathrm{O}-\mathrm{CH}_{2}-\mathrm{CH}_{2}\right)$; 1.57-1.29 (m, 18H, - $\left.\mathrm{CH}_{2}-\right)$; $0.91\left(\mathrm{t}, 3 \mathrm{H}, 6.6 \mathrm{~Hz},-\mathrm{CH}_{3}\right) ;{ }^{13} \mathrm{C} \mathrm{NMR}\left(400 \mathrm{MHz}, \mathrm{CDCl}_{3}\right) \delta 161.78,147.57$, $143.31,134.84,132.46,131.36,126.09,118.24,114.53,68.37,31.93,29.69,29.60,29.38,29.16,26.04$, 22.71, 14.15. MS (TOF-ES) $475.3\left(\mathrm{MNa}^{+}\right)$. 


\section{Synthesis and characterization of 2.}

Compound 2 was synthesized by a procedure analogous to 1, using 4-formyl farnesylbenzoate (4FF) with excess pyrrole in the presence of a Lewis acid to form the corresponding dipyrromethane, followed by oxidation with DDQ and addition of $\mathrm{BF}_{3}$-etherate. The 4-formyl farnesyl benzoate (4FF) was prepared separately by adaptation of the literature procedure ${ }^{41}$ and involved esterification of commercially available 4-carboxybenzaldehyde with trans, trans-farnesol.

\section{Synthesis of 4FF.}

Farnesol (0.45 g, 2mmol) was added to a cooled (ice-bath), stirred solution of 4-carboxybenzaldehyde (0.3 g, 2mmol) in dry DCM/THF (9:1), followed by DCC (0.41 g, 2mmol) and a catalytic amount of DMAP. The ice-bath was removed and the resulting mixture was left to warm up to room temperature and stirred overnight under argon. The resulting precipitate was filtered off and the filtrate evaporated to give a white waxy-solid. This solid was purified by column chromatography (silica gel, DCM, Rf 0.54 ) to give $4 \mathrm{FF}$ as a clear oil. Yield $0.3 \mathrm{~g}(42 \%):{ }^{1} \mathrm{H}$ NMR $\left(400 \mathrm{MHz}, \mathrm{CDCl}_{3}\right) \delta 10.13(\mathrm{~s}, 1 \mathrm{H}, \mathrm{CHO}) ; 8.22$ $(\mathrm{d}, \mathrm{J}=8.2,2 \mathrm{H}$, phenyl-H); $7.96(\mathrm{~d}, \mathrm{~J}=8.2,2 \mathrm{H}$, phenyl-H); 5.52-5.48 (m, 1H, vinyl-H); 5.15-5.07 (m, 2H, vinyl-H); 4.90 (d, J=7.1, $\left.\mathrm{CH}_{2}-\mathrm{O}\right) ; 2.19-1.97\left(\mathrm{~m}, 8 \mathrm{H},-\mathrm{CH}_{2}-\right) ; 1.80\left(\mathrm{~s}, 3 \mathrm{H}, \mathrm{CH}_{3}\right), 1.69$ (s, 3H, $\left.\mathrm{CH}_{3}\right) ; 1.62$ $\left(\mathrm{s}, 3 \mathrm{H}, \mathrm{CH}_{3}\right), 1.60\left(\mathrm{~s}, 3 \mathrm{H}, \mathrm{CH}_{3}\right) ;{ }^{13} \mathrm{C} \mathrm{NMR}\left(400 \mathrm{MHz}, \mathrm{CDCl}_{3}\right) \delta 191.72,165.61,143.04,139.04,135.55$, $131.35,130.21,129.48,124.27,123.50,117.95,62.47,39.68,39.54,26.69,26.12,25.17,17.69,16.59$, 16.05; MS (ES) $355.23\left(\mathrm{MH}^{+}\right)$.

\section{Synthesis of 2}

The aldehyde 4FF (0.2 g, $0.56 \mathrm{mmol})$ was dissolved in excess freshly distilled pyrrole $(1 \mathrm{ml}, 0.014$ mol) and the resulting solution degassed by gently bubbling argon through the solution for $c a .5 \mathrm{~min}$. before the addition of boron trifluoride etherate $(24 \mu 1)$. The resulting mixture was stirred for $30 \mathrm{~min}$. quenched by the addition of aqueous $\mathrm{NaOH}$ solution $(2 \mathrm{ml}, 0.1 \mathrm{M})$ and extracted with $\mathrm{DCM}(2 \times 5 \mathrm{ml})$. 
The combined organic extracts were washed with water with the addition of small amounts of brine, separated, dried over $\mathrm{Na}_{2} \mathrm{SO}_{4}$ and the solvent taken off using a rotary evaporator. The excess pyrrole was removed using high vacuum to give the dipyrromethane as a green viscous oil. TLC (silica gel, DCM) confirmed consumption of the aldehyde (4FF) and the presence of the dipyrromethane, visible as a red/purple spot on exposure of the TLC plate to bromine vapour. The crude dipyrromethane was dissolved in toluene $(10 \mathrm{ml})$, DDQ $(0.1 \mathrm{~g}, 0.46 \mathrm{mmol})$ was added and the reaction mixture stirred at room temperature shielded from light. Following 5 minutes of stirring, TLC (silica gel, 5\% ethyl acetate/DCM $)$ showed the consumption of the dipyrromethane at which point excess triethylamine $(0.5$ $\mathrm{ml}, 3.62 \mathrm{mmol})$ was added followed immediately by boron trifluoride etherate $\left(320 \mu \mathrm{l}\right.$ of neat $\mathrm{BF}_{3^{-}}$ etherate, $2.58 \mathrm{mmol})$. The reaction mixture was stirred at room temperature for $1 \mathrm{~h}$, washed with water, dried $\left(\mathrm{Na}_{2} \mathrm{SO}_{4}\right)$ and evaporated to give a dark-green viscous oil. This was purified by column chromatography (silica gel, 5\% ethyl acetate,DCM, Rf 0.68) to give 2 as a red-orange sticky solid. Yield $55 \mathrm{mg}(19 \%):{ }^{1} \mathrm{H}$ NMR (400 MHz, $\left.\mathrm{CDCl}_{3}\right) \delta \quad 8.23$ (d, J=8.16, 2H, phenyl-H); 7.99 (s, 2H, pyrrole-H); $7.66(\mathrm{~d}, \mathrm{~J}=8.16,2 \mathrm{H}$, phenyl-H); $6.91(\mathrm{~d}, 2 \mathrm{H}, \mathrm{J}=4$, pyrrole-H); 6.59 (m, 2H, pyrrole-H); 5.54-5.50 (m, 1H, vinyl-H); 5.15-5.09 (m, 2H, vinyl-H); 4.93 (d, J=7.1, $\left.\mathrm{CH}_{2}-\mathrm{O}\right) ; 2.20-1.98\left(\mathrm{~m}, 8 \mathrm{H},-\mathrm{CH}_{2}-\right) ; 1.82(\mathrm{~s}$, $\left.3 \mathrm{H}, \mathrm{CH}_{3}\right), 1.69\left(\mathrm{~s}, 3 \mathrm{H}, \mathrm{CH}_{3}\right) ; 1.63\left(\mathrm{~s}, 3 \mathrm{H}, \mathrm{CH}_{3}\right), 1.61\left(\mathrm{~s}, 3 \mathrm{H}, \mathrm{CH}_{3}\right) ;{ }^{13} \mathrm{C} \mathrm{NMR}\left(400 \mathrm{MHz}, \mathrm{CDCl}_{3}\right)$ $\delta 165.67,161.71,147.26,143.55,143.04,139.04,134.88,135.54,131.35,131.11130 .51,129.69$, $124.27,123.50,118.23,117.95,62.41,39.88,39.55,26.69,26.12,25.17,17.70,16.59,16.05$; MS (TOFES) $549.4\left(\mathrm{MNa}^{+}\right)$.

\section{Viscosity measurements (rheometer)}

The viscosity of methanol/glycerol mixtures was measured using an advanced rheometric expansion system rheometer $(\mathrm{ARES})^{3}$ equipped with a force-rebalance transducer (torque range, $4 \times 10^{-8}-1 \times 10^{-3}$ $\mathrm{Nm}$ ). A double-walled Couette-type rheometric tool was used for the measurements (cup outer radius, $17.00 \mathrm{~mm}$; cup inner radius, $13.98 \mathrm{~mm}$; bob outer radius $16.00 \mathrm{~mm}$; bob inner radius, $14.75 \mathrm{~mm}$, 
resulting in $1 \mathrm{~mm}$ external gap and $0.77 \mathrm{~mm}$ inner gap. The temperature was kept at $(25.0 \pm 0.1)^{\circ} \mathrm{C}$ with a bath circulator (Julabo F33). Each measurement used ca. $7 \mathrm{ml}$ of sample. The viscosity was measured as a function of shear rate in the range from 1 to $100 \mathrm{~s}^{-1}$ and was found to be constant.

\section{Cell manipulation}

The human ovarian carcinoma cell line SK-OV-3 was obtained from the European Collection of Cell Cultures (ECACC). Cells were cultured in Dulbecco's modified Eagle's medium (DMEM) with 10\% foetal calf serum, penicillin and streptomycin antibiotics and passaged when $70-90 \%$ confluent in 75 $\mathrm{cm}^{3}$ flasks grown at $37^{\circ} \mathrm{C}$ in $5 \% \mathrm{CO}_{2}$. For imaging, SK-OV-3 cells were seeded at $10^{4}$ cells/well in 0.2 $\mathrm{ml}$ of culture medium in untreated 8-well coverglass chambers (Lab-TekTM, Nunc) and allowed to grow to confluence for $24 \mathrm{~h}$. The culture media was replaced with the culture medium containing $\mathbf{1}$ or $\mathbf{2}$ and incubated for 30 min. Following incubation, the chambers were washed twice with phosphate buffered saline prior to imaging.

\section{Fluorescence lifetime and FLIM}

Fluorescence lifetime and FLIM measurements were performed by coupling a pulsed diode laser (PLP-10 470, Hamamatsu, $\lambda=467 \mathrm{~nm}$, repetition rate $=20 \mathrm{MHz}$, pulse duration $\sim 90 \mathrm{ps}$ ) into an inverted confocal microscope (Leica TCS SP2). The fluorescence was separated from the excitation beam by a chromatic reflector before passing through a bandpass filter $(525 \pm 25 \mathrm{~nm})$ placed before a cooled photomultiplier detector (PMC-100-01, Becker \& Hickl, based on a Hamamatsu H5772P-01). Time-correlated single photon counting was performed using a Becker \& Hickl SPC-830 TCSPC card in a PC. Acquisition times were $300 \mathrm{~s}$ with a line scan rate of $400 \mathrm{~Hz}$. A $63 \times$ water immersion objective $(\mathrm{NA}=1.2)$ was used for all measurements. Pixel fits to the lifetime data were performed using SPCImage (Becker \& Hickl) and TRI2 ${ }^{42,43}$ software.

\section{Fluorescence anisotropy measurements}


Anisotropy measurements were made by placing a polarizing beamsplitter cube into the detection arm with orthogonally polarized components of the fluorescence being detected by two cooled PMC-100-01 photomultiplier detectors simultaneously. The output of the photomultipliers was fed into a router (Becker \& Hickl HRT-41) prior to the TCSPC detection using the SPC-830 hardware. Thus, fluorescence intensity decay curves for fluorescence polarized parallel and perpendicularly to the polarization vector of the linearly polarized incident radiation were recorded simultaneously. The Gfactor (which accounts for differences in detection efficiency for the orthogonal polarization components) for the detection was determined to be $\sim 1.1$ by tail matching of the parallel and perpendicular decays of FITC in solution. Confocal microscopy using $488 \mathrm{~nm}$ continuous wave excitation was used to obtain images of the cells. Fluorescence decays for each polarization were extracted from relevant regions of the cell images and analysed using TRI2 software and the timeresolved anisotropy was calculated according to equation 6 . The photon count rate was kept well below $1 \%$ of the laser repetition rate, effectively rendering the possibility of cross-channel pile-up in the detection negligible. $^{44}$

A low value of the measured initial anisotropy in microscopy experiments does not necessarily indicate that there is an $r_{\infty}$ value. It is well known that the use of a high numerical aperture objective reduces the maximum measured value, ${ }^{45}$ so that the use of the $63 \times \mathrm{NA}=1.2$ water immersion objective will reduce the recovered value of $r_{0}$ to below 0.4 .

\section{Results and Discussion}

\section{Photophysical characterisation}

We have previously reported that $\mathbf{1}$ can act as a fluorescent molecular rotor, with emission spectrum and fluorescence lifetime sensitive to the ambient viscosity. ${ }^{24}$ The corresponding changes for 2 are shown in Figure $1(a, b)$. The shape of the emission spectrum and the wavelength of the peak emission are invariant as a function of viscosity with the peak of the emission at $530 \mathrm{~nm}$. It can be seen that 
increasing the viscosity increases the emission intensity and fluorescence lifetime of 2, which is consistent with the Förster-Hoffmann equation (3). Similar changes have been observed previously for 1. $^{24}$

The values of $\Phi_{f}, k_{r}$ and $k_{n r}$ for dyes $\mathbf{1}$ and $\mathbf{2}$ as a function of viscosity in methanol/glycerol solutions are shown in Figure 2 (a) and (b). The values were calculated from the experimentally measured $\Phi_{f}$, and fluorescence lifetime, $\tau_{f}$, via equations 2 and 4 . In non-viscous pure methanol $\Phi_{f}=0.06$ for both 1 and 2 which is consistent with literature values for phenyl-substituted BODIPY dyes. ${ }^{46}$ For both $\mathbf{1}$ and $\mathbf{2}$ the quantum yield increases significantly as the viscosity of the solution increases, as expected from equation 1. Interestingly the value of $k_{r}$ remains largely constant as a function of the viscosity (the slight increase may be due to an increase in the refractive index ${ }^{47}$ ) while the value of $k_{n r}$ decreases sharply with increasing viscosity up to $\sim 300 \mathrm{cP}$, Figure 2 (a) and (b) and Table 1 . These data indicate that the increase in $\Phi$ with increasing viscosity is due to the suppression of non-radiative processes, consistent with the excited state deactivation mechanism of phenyl-substituted BODIPYs proposed earlier. ${ }^{46}$ Thus, by hindering intramolecular rotation, the viscous environment prevents access to a lower lying dark state characterised by a puckered geometry in which the phenyl ring is rotated into the plane of the BODIPY ring. The most stable conformation, from which the radiative decay takes place is characterised by a $60^{\circ}$ twist of the phenyl group vs. the BODIPY plane. We note that in the most viscous solution studied ( $95 \%$ glycerol, $\eta=950 \mathrm{cP}$ ) the non-radiative processes are completely suppressed and the fluorescence quantum yield for both $\mathbf{1}$ and $\mathbf{2}$ is 1 (Figure 2). 

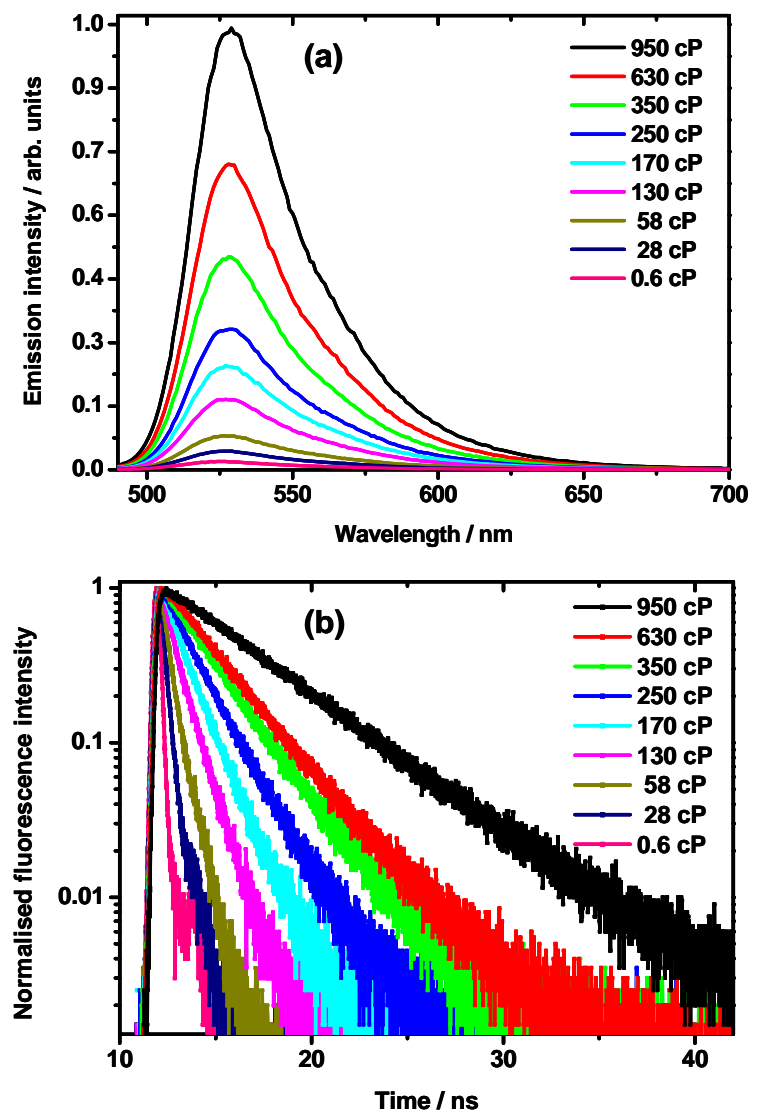

Figure 1. The emission spectra (a) and fluorescence decays (b) of 2 recorded as a function of viscosity in methanol/glycerol solutions. Both the fluorescence intensity and the fluorescence lifetime decrease as the viscosity decreases. 

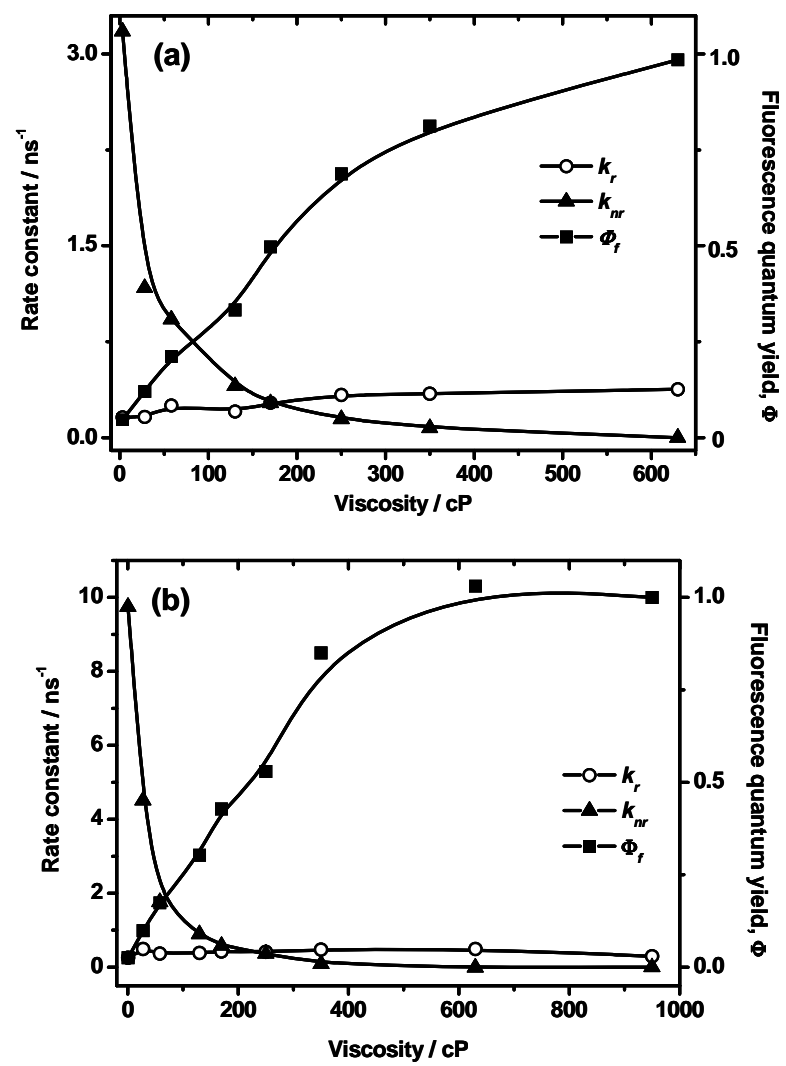

Figure 2. The fluorescence quantum yield $\left(\Phi_{\mathrm{f}}\right)$, and radiative $\left(k_{r}\right)$ and non-radiative $\left(k_{n r}\right)$ rate constants, obtained as a function of viscosity in methanol/glycerol solutions of $\mathbf{1}$ (a) and $\mathbf{2}$ (b).

\begin{tabular}{|c|c|c|c|c|c|c|c|}
\hline & & \multicolumn{3}{|c|}{ Dye 1- $\mathrm{C}_{12}$ chain } & \multicolumn{3}{c|}{ Dye 2 - farnesyl chain } \\
\cline { 3 - 8 } \begin{tabular}{c} 
Glycerol \\
\cline { 3 - 8 }
\end{tabular} & $\begin{array}{c}\text { Viscosity } \\
\mathrm{cP}\end{array}$ & $\begin{array}{c}\text { Fluorescence } \\
\text { quantum } \\
\text { yield } / \Phi_{f}\end{array}$ & $k_{r} / \mathrm{ns}^{-1}$ & $k_{n r} / \mathrm{ns}^{-1}$ & $\begin{array}{c}\text { Fluorescence } \\
\text { quantum } \\
\text { yield } / \Phi_{f}\end{array}$ & $k_{r} / \mathrm{ns}^{-1}$ & $k_{n r} / \mathrm{ns}^{-1}$ \\
\hline 100 & 950 & - & - & - & 1.00 & 0.29 & 0.00 \\
\hline 95 & 630 & 1.00 & 0.38 & 0.00 & 1.00 & 0.49 & 0.00 \\
\hline 90 & 350 & 0.83 & 0.34 & 0.07 & 0.85 & 0.47 & 0.08 \\
\hline 85 & 250 & 0.70 & 0.33 & 0.14 & 0.53 & 0.41 & 0.37 \\
\hline 80 & 170 & 0.50 & 0.27 & 0.27 & 0.43 & 0.43 & 0.57 \\
\hline 75 & 130 & 0.34 & 0.20 & 0.40 & 0.30 & 0.39 & 0.89 \\
\hline 70 & 58 & 0.21 & 0.25 & 0.92 & 0.17 & 0.37 & 1.76 \\
\hline 60 & 28 & 0.12 & 0.16 & 1.17 & 0.10 & 0.49 & 4.51 \\
\hline 50 & 3 & 0.05 & 0.16 & 3.17 & 0.03 & 0.25 & 9.75 \\
\hline
\end{tabular}

Table 1. Values for the fluorescence quantum yield $\left(\Phi_{\mathrm{f}}\right)$, and radiative $\left(k_{r}\right)$ and non-radiative $\left(k_{n r}\right)$ rate constants, obtained as a function of viscosity in methanol/glycerol solutions of dye $\mathbf{1}$ and dye $\mathbf{2}$ (errors are estimated to be $\pm 10 \%$ for fluorescence quantum yield and rate constants). 


\section{Fluorescence lifetime measurements of BODIPY in methanol/glycerol}

Fluorescence decays measured for 2 at increasing viscosity in methanol/glycerol mixtures are shown in Figure 1(b). The fluorescence lifetime varies markedly as a function of viscosity increasing from $\sim 100 \mathrm{ps}$ at $0.6 \mathrm{cP}$ to $3.4 \mathrm{~ns}$ at $950 \mathrm{cP}$. The plots of fluorescence lifetimes $v s$ viscosity for $\mathbf{1}$ and 2 in methanol/glycerol solutions are shown in Figure 3. From equation 5 it is expected that the log-log plot should yield a linear dependence between log-lifetime and log-viscosity. Indeed, this is evident at viscosities above $\sim 20 \mathrm{cP}$ for both dyes, giving gradients $\alpha$ of $\sim 0.5 \pm 0.1$ and $\sim 0.75 \pm 0.1$ for $\mathbf{1}$ and 2 , respectively. These compare well with the value of $2 / 3$ predicted by Förster and Hoffmann. ${ }^{34}$ We find that below $20 \mathrm{cP}$ the fluorescence lifetime is minimally variant. Although the Förster-Hoffmann equation predicts linearity over several orders of magnitude of viscosity, we believe that the apparent discrepancy at low viscosity $(<20 \mathrm{cP})$ for $\mathbf{1}$ and $\mathbf{2}$ is due to geometry-dependent photophysics. The viscosity-dependent rotational barrier for the phenyl group ${ }^{46}$ from the most stable conformation $\left(\sim 60^{\circ}\right.$ relative to the BODIPY unit) to the metastable conformation (in the plane of the BODIPY unit) becomes insignificant at viscosities $<20 \mathrm{cP}$ and can be easily overcome at room temperature.

Importantly, values for the fluorescence lifetime at a given viscosity, along with the intercept of the axis are markedly lower in the case of the farnesyl-substituted molecule. From equation 5 the intercept of the log-log plot gives the value $\log \frac{z}{k_{r}}$ and as such a lower value for the intercept corresponds to a higher value for $k_{r}$. This is consistent with our earlier observations that $k_{r}(\mathbf{1})$ is greater than $k_{r}(\mathbf{2})$ (Figure 2). 


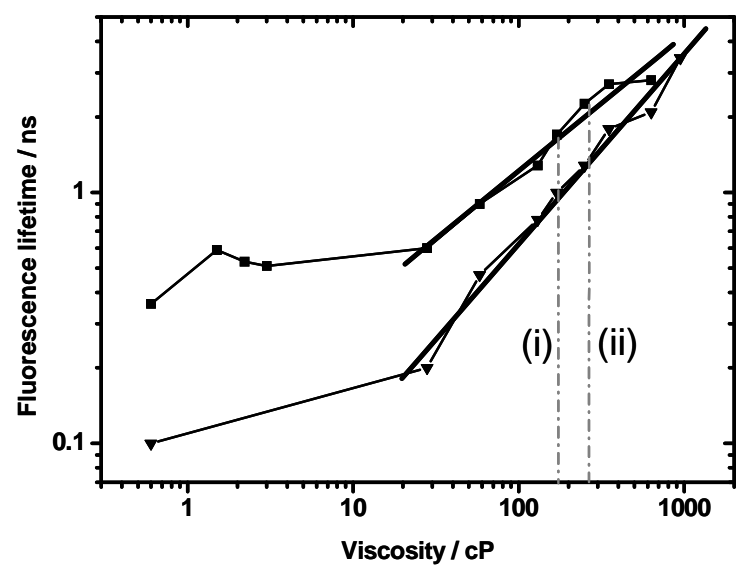

Figure 3. Log fluorescence lifetime $\left(\tau_{f}\right) v s \log$ viscosity $(\eta)$ for $\mathbf{1}$ (filled squares) and $\mathbf{2}$ (filled triangles). Above $\eta \sim 20 \mathrm{cP}$ there is a linear dependence of $\log \tau_{f}$ vs $\log \eta$. The vertical dashed lines are guides for the eye and correspond to the intracellular fluorescence lifetimes in (i) puncta and (ii) cytoplasmic regions.

We aim to use the fluorescence lifetime vs. viscosity calibration plots obtained in methanol/glycerol mixtures (Figure 3) to determine the viscosity distribution in cells via FLIM. However, the fluorescence lifetime can be influenced by several environmental parameters apart from viscosity, such as refractive index, polarity, $\mathrm{pH}$, or chemical and physical quenching processes. ${ }^{30}$ It is well known that the spectral characteristics of unmodified BODIPY chromophores, including lifetimes, are largely independent on the solution $\mathrm{pH}$ and polarity of the solvent. ${ }^{48,49}$ The quenching of BODIPYs with protein components, e.g. tryptophan (Stern Volmer quenching constant, $\mathrm{K}$, of $\left.15 \mathrm{M}^{-1}\right)$, tyrosine $\left(\mathrm{K}=14 \mathrm{M}^{-1}\right)$ phenylalanine $\left(\mathrm{K}=1.4 \mathrm{M}^{-1}\right)$ has been investigated ${ }^{48}$ and this study concluded that such quenching requires a high concentration of the quencher and leads to non-monoexponential excited state decays of BODIPY, which is not observed in our study for either $\mathbf{1}$ or $\mathbf{2}$.

We have also confirmed that the refractive index has only a minor effect on the fluorescence lifetime of the BODIPY chromophore, by analyzing the fluorescence lifetimes of a structurally similar BODIPY dye, collected over a wide range of refractive indices, $n$, by López Arbeloa et al. ${ }^{50}$ The BODIPY 
fluorescence lifetimes measured in a range of solvent were plotted as a function of $n^{2}(n \sim 1.3-1.54)$ using raw data from reference ${ }^{50}$ (Figure 4). The data fit well with a linear function consistent with the Strickler-Berg formula. ${ }^{51}$ The data confirm that the change in fluorescence lifetime over a very wide range of $n^{2}$ (greater than the change in $n$ for methanol/glycerol solutions with viscosity ranging from 0.6 cP to $950 \mathrm{cP}$ ) is $\sim 20 \%$ which is around an order of magnitude less than the fluorescence lifetime change due to viscosity for our dyes 1 and 2. Hence, the contribution of $n$ in our FLIM measurements of intracellular viscosity is small.

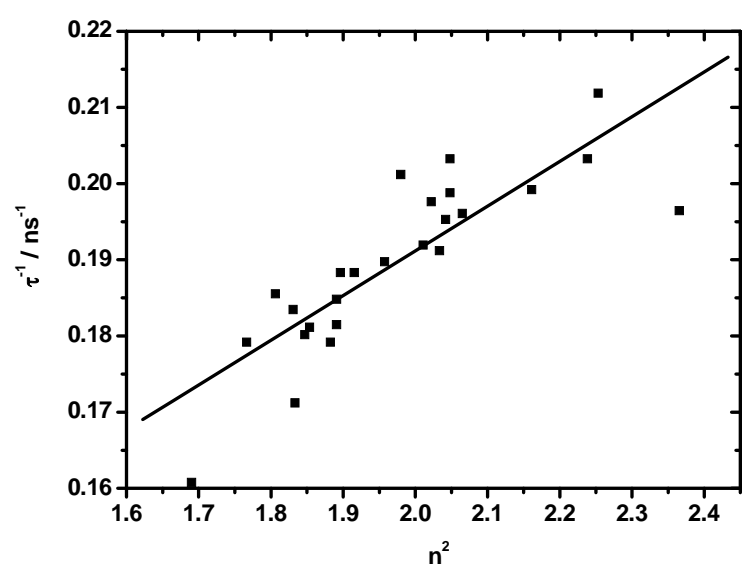

Figure 4. The plot of fluorescence lifetimes vs refractive index of the solvent for structurally related viscosity-independent BODIPY dye. The raw data are taken from reference ${ }^{50}$. The data fit well with a linear function consistent with the Strickler-Berg formula. ${ }^{51}$

\section{Fluorescence microscopy and FLIM}

Both 1 and 2 are readily taken up by SK-OV-3 cells. The intracellular distribution pattern for both dyes is shown in Figure 5 (a) and (c) and is consistent with that previously reported for $1 .{ }^{24}$ In addition to the bright punctate distribution of $\mathbf{1}$ and $\mathbf{2}$ in cells, we also observed regions of lower fluorescence intensity in the cell cytosol. Due to the hydrophobic nature of $\mathbf{1}$ and $\mathbf{2}$ and the presence of the long tails which render them membrane soluble, we expect both $\mathbf{1}$ and $\mathbf{2}$ to target the membrane domains of 
intracellular organelles. We note that the membrane solubility of $\mathbf{2}$ is expected to be 10 times greater than that of $\mathbf{1}$ due to the nature of the farnesyl chain. ${ }^{52}$
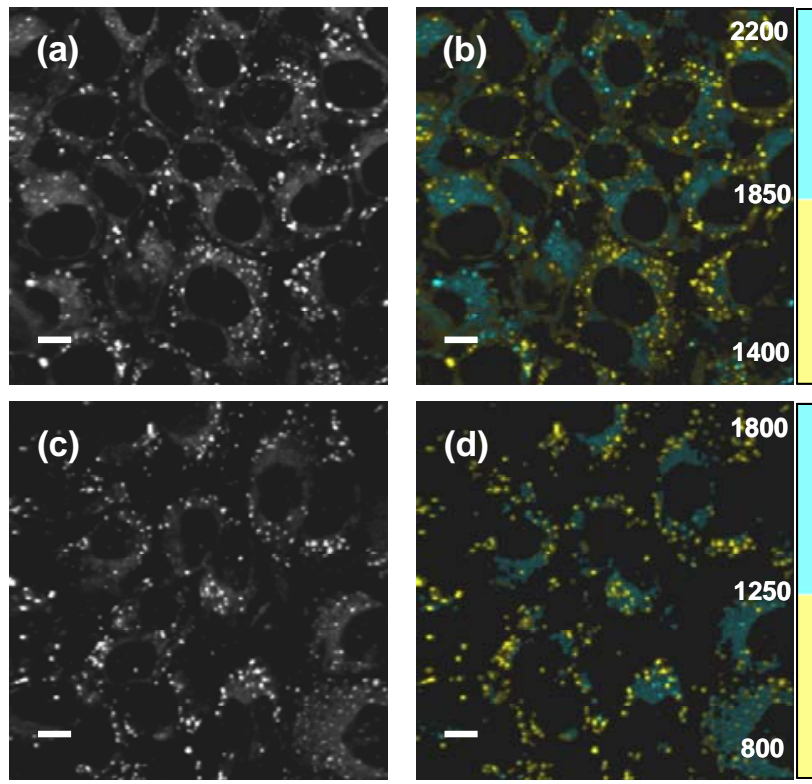